\title{
Inducing Cooperation for Optimal Coexistence in Cognitive Radio Networks: A Game Theoretic Approach
}

\author{
Muhammad Faisal Amjad Mainak Chatterjee Cliff C. Zou \\ Department of Electrical Engineering and Computer Science \\ University of Central Florida, Orlando FL 32816-2362 \\ \{faisal,mainak,czou\}@eecs.ucf.edu
}

\begin{abstract}
Current coexistence protocols employed for contention by collocated Cognitive Radio Networks (CRN), such as the IEEE 802.22 WRAN, assume that the contending networks do not have any preference over the set of available channels. Having channels with different quality parameters can lead to an imbalance in contention for disparate channels, degraded quality of service and an overall inefficient utilization of spectrum resources. In this paper, we analyze this situation from a game theoretic perspective and model coexistence of CRNs as a noncooperative, repeated general-sum game with perfect information. We demonstrate that due to the possibility of its centralized as well as a distributed implementation, the correlated equilibrium is a practical solution for the problems of inefficiency and unfairness of Nash Equilibria. It not only induces voluntary cooperation among non-cooperative CRNs and results in optimum spectrum utilization but also results in an egalitarian equilibrium which maximizes the minimum payoff for every CRN.
\end{abstract}

\section{INTRODUCTION}

Studies on spectrum utilization have shown that static allocation of the spectrum has resulted in severe underutilization of this scarce resource, even as low as 14\% [1]. With the proliferation of devices that rely on wireless access to the internet, the demand for wireless spectrum bands is everincreasing. This wide gap in the demand and supply of wireless spectrum resource forced regulatory bodies such as the FCC to allow un-licensed access to spectrum bands, also referred to as the TV white spaces, otherwise licensed to the Primary Users (PUs) in an opportunistic and non-interfering basis [2]. This has given rise to a challenging as well as an exciting type of networks called the Cognitive Radio Networks (CRNs). Deployment of Cognitive Radio technology as a last-mile connectivity option has already begun in parts of the world.

Dynamic Spectrum Access (DSA) allows CRNs to ensure that their use of spectrum does not cause interference to PUs and that all spectrum opportunities are utilized to the maximum. IEEE 802.22 wireless regional area network (WRANs) [3] is an example of CRNs in which the base station controls all the operation of the CRN including the choice of spectrum bands for communication. However, there may be many CRNs collocated in a region all of whom compete for access to the available channels, a situation called self coexistence in the context of CRNs. Most coexistence protocols do not take into consideration the fact that these channels can be heterogeneous in the sense that they can vary in their characteristics and quality such as SNR or bandwidth. Without any mechanism to enforce fairness in accessing varying quality channels, ensuring coexistence with minimal contention and efficient spectrum utilization for CRNs is likely to become a very difficult task.

In this paper, we model heterogeneous spectrum sharing in CRNs as a repeated non-cooperative anti-coordination game where the payoff for every player (i.e., CRN) in the game is determined by the quality of the spectrum band to which it was able to gain access. Game theory has been applied to numerous areas of research involving conflict, competition and cooperation among multi-agents systems including wireless communications. Online databases $[4,5]$ can be accessed by CRNs to gain information about licensed PUs operating in a given region. Furthermore, the FCC requires $\mathrm{CRNs}$ to periodically access these online databases for up-to-date information about PU activity in their areas of operation. Therefore, the amount of PU activity, bandwidth and SNR which, for the purpose of this paper collectively determine a channel's quality can be learnt / measured over a period of time. Due to the fact that all contending CRNs are collocated in a given region, it is reasonable to assume a channel's quality to be common knowledge. Being rational about their choices, every player has a clear preference of selecting the best available channels before the start of every time slot. However, if every player tries to access the best available channel, it will result in a collision and the spectrum opportunity being wasted. Players that eventually gain access to higher quality channels will gain higher payoffs as compared to the players that end up with lower quality channels.

To propose fair and efficient strategies for utilization of the spectrum resources, we formulate an anti-coordination game in which we derive the game's pure (PSNE) and mixed strategy Nash Equilibria (MSNE) along with its Correlated equilibrium (CE). We demonstrate how the traditional solution concepts of Nash Equilibria (NE) are either inefficient or unfair while the correlated equilibrium is optimal and fair. We also demonstrate how CE can be achieved in a 2-player as well as an $N$-player game with centralized as well as a distributed approach using linear optimization and No-Regret learning algorithms respectively.

\section{RELATED WORK}

Correlated equilibrium has been employed in [6] for a P2P file sharing non-cooperative game to jointly optimize players' expected delays in downloading files. Not uploading files for others causes an increase in file download time for all players which in turn forces even the non-cooperative players to 
cooperate. The authors of [7] tackle the self-coexistence problem of finding a mechanism that achieves a minimum number of wasted time slots for every collocated CRN to find an empty spectrum band for communications. To do so, they employ a distributed modified minority game under incomplete information assumption. Authors of [8] employ punishment strategies in a Gaussian interference game for a one shot game as well as an infinite horizon repeated game to enforce cooperation. Spectrum sharing is however considered within the context of a single CRN. Evolutionary game theory is applied in [9] to solve the problem in a joint context of spectrum sensing and sharing within a single CRN. Multiple SUs are assumed to be competing for unlicensed access to a single channel. SUs are considered to have half-duplex devices so they cannot sense the channel and access it at the same time.

Utility graph coloring is used to address the problem of selfcoexistence in CRNs in [10]. Allocation of spectrum for multiple overlapping CRNs is done using graph coloring in order to minimize interference and maximize spectrum utilization using a combination of aggregation, fragmentation of channel carriers, broadcast messages and contention resolution. The authors of [11] achieve correlated equilibrium with the help of No-regret learning algorithm to address the problem of network congestion when a number of SUs within a single CRN contend for access to channels using a CSMA type MAC protocol. They model interactions of SUs within the $\mathrm{CRN}$ as a prisoner's dilemma game in which payoffs for the players are based on aggressive or non-aggressive transmission strategies after gaining access to idle channels.

\section{SYTEM MODEL}

We consider a region where IEEE 802.22 WRAN based CRNs represented by the set of $N=\{1,2, \ldots, n\}$ players are collocated and contend for secondary access to the licensed spectrum bands. The set of Television whitespace (TVWS) channels available for secondary access by the contending CRNs is represented as $K=\{1,2, \ldots, k\}$ channels. The spectrum consists of channels that differ from each other due to various network parameters such as noise, bandwidth or even availability. These differences make the spectrum heterogeneous in nature with channels considered to have some 'quality' parameter determined by the payoff that a CRN may achieve ${ }^{1}$ if it is able to gain access to that channel. CRNs need to gain access to a channel in every time slot. A time slot is also treated as a stage in the spectrum sharing game.

All CRNs are independent as they do not share a common goal and therefore do not cooperate with each other. A given time slot's spectrum opportunity that arises due to the absence of its primary user may result in a collision and therefore be wasted if two or more CRNs select the same channel for access. Since all CRNs are collocated, a channel's SNR, available bandwidth and PU's activity on their licensed channels can be measured by all CRNs. Furthermore, sensing of PU's spectrum allocation/activity is mandated by the FCC

\footnotetext{
${ }^{1}$ We use the terms utility and payoff interchangeably.
}

for CRNs [2] and is also publically available through online databases $[4,5]$. Therefore, considering a channel's quality to be common knowledge is reasonable. In subsequent section, we show that our proposed spectrum sharing game can be implemented solely on the basis of a CRN's own payoff observations.

\section{CORRELATED EQUILIBRIUM FOR SPECTRUM SHARING GAME}

In this section, we first present the formulation of our proposed spectrum sharing game, followed by the derivation of pure and mixed strategy Nash Equilibria. Next we introduce the concept of Correlated Equilibrium (CE) and demonstrate how $\mathrm{CE}$ can be achieved in a centralized implementation for a 2-player game using linear optimization. We also demonstrate that $\mathrm{CE}$ can be achieved in a distributed manner for an $N-$ player game using a learning algorithm called No-Regret (NR) learning [14]. Using these concepts we model the problem of self-coexistence and heterogeneous spectrum sharing in the following subsections as an anti-coordination game framework. The game is a non-cooperative repeated game with perfect information because:

- CRNs compete for the best channels available in the spectrum band and are interested only in maximizing their own utility. Therefore, CRNs are not bound to cooperate with each other.

- Utilities are common knowledge since the quality of various network parameters can be measured by every CRN. Also, it is reasonable to assume that every CRN can tell which channels other CRNs were able to gain access to in the past hence they know other CRNs' payoffs.

\section{A. Game Formulation}

The spectrum sharing anti-coordination game presented in this paper is represented as $\mathcal{G}=\langle N,(A),(U)\rangle$, where players in the game are CRNs represented by $N$. Every player in the game has the same action/strategy space represented by $A=$ $\left\{a_{1}, a_{2} \ldots, a_{k}\right\}$ where the strategy $a_{k}$ means selecting channel $k$ for communication during the next time slot. The CRNs gain a specific payoff when they are successful in utilizing a spectrum opportunity in a channel. The payoff for players playing strategies $a_{k}$ and $a_{j}$ respectively, when competing against each other for access to channels is denoted by the ordered pair $u\left(a_{k}, a_{j}\right) \in U$ and is a function of an individual channel's quality given by:

$$
u\left(a_{k}, a_{j}\right)= \begin{cases}\left(u_{k}, u_{j}\right), & k \neq j \\ (0,0), & k=j\end{cases}
$$

where the first element $u_{k}$ of the ordered pair $u\left(a_{k}, a_{j}\right)$ represents the payoff for the player that selected channel $k$ and the second element $u_{j}$ for the player selecting channel $j$. For the sake of ease in analysis, we assume that $u_{k}>u_{j}$. The game represented by (1) can also be represented in strategic 
Table I: Strategic form representation of a 2-player anticoordination game with strategies $a_{k}$ and $a_{j}$. Players receive a payoff of 0 if both select the same strategy.

\begin{tabular}{|c|c|c|}
\cline { 2 - 3 } \multicolumn{1}{c|}{} & $\boldsymbol{a}_{\boldsymbol{k}}$ & $\boldsymbol{a}_{\boldsymbol{i}}$ \\
\hline $\boldsymbol{a}_{\boldsymbol{k}}$ & $(0,0)$ & $\left(u_{k}, u_{j}\right)$ \\
\hline $\boldsymbol{a}_{\boldsymbol{j}}$ & $\left(u_{j}, u_{k}\right)$ & $(0,0)$ \\
\hline
\end{tabular}

form as table I, which shows the payoffs for two players selecting channels $k$ or $j$. Since $u_{k}>u_{j}$, it is in every CRN's interest to choose channel $k$ instead of channel $j$ for a larger payoff. However, when the players select the same channel it results in a collision, the spectrum opportunity being wasted and both player end up with a payoff of 0 . On the other hand, if both players select different channels then their payoffs reflect the quality of the channel to which they are able to gain access. This game is the reverse of the classic Battle of the Sexes game and is classified as an anti-coordination game where it is in both players' interest not to end up selecting the same strategy, as shown in table I.

\section{B. Pure and Mixed Strategy Nash Equilibria for the Spectrum Sharing Game}

In this subsection we derive the solution concepts in the form of pure (PSNE) as well as the mixed strategy Nash equilibria (MSNE) for our spectrum sharing anti-coordination game.

Definition 1: The pure strategy Nash Equilibrium [12] of the spectrum sharing game is an action profile $a^{*} \in A$ of actions, such that

$$
u\left(a_{i}^{*}, a_{-i}^{*}\right) \gtrsim u\left(a_{i}, a_{-i}^{*}\right), \quad \forall i \in K
$$

where $\gtrsim$ is a preference relation over utilities of strategies $a_{i}^{*}$ and $a_{i}$. The above definition means that for $a_{i}^{*}$ to be a pure strategy NE, it must satisfy the condition that no player $i$ has another strategy that yields a higher payoff than the one for playing $a_{i}^{*}$ given that every other player plays their equilibrium strategy $a_{-i}^{*}$.

Lemma 1: Strategy pairs $\left(a_{k}, a_{j}\right)$ and $\left(a_{j}, a_{k}\right)$ are pure strategy NE of the anti-coordination game.

Proof: Assume player 1 to be the row player and player 2 to be the column player in table I. From (1) it follows that both $u_{k}$ and $u_{j}$ are positive values and therefore the payoffs for strategy pairs $\left(a_{k}, a_{j}\right)$ and $\left(a_{j}, a_{k}\right)$ are greater than the payoffs for strategy pairs $\left(a_{k}, a_{k}\right)$ and $\left(a_{j}, a_{j}\right)$. Consider the payoff for strategy pair $\left(a_{k}, a_{j}\right)$ from table I. Given that the player playing strategy $a_{j}$ continues to play this strategy, then from definition 1 for a Nash Equilibrium, it follows that the player playing strategy $a_{k}$ does not have any incentive to change its choice to $a_{j}$, i.e., it will receive a smaller payoff of 0 if it switched to $a_{j}$. Therefore, $\left(a_{k}, a_{j}\right)$ is a PSNE. Similarly, strategy pair $\left(a_{j}, a_{k}\right)$ is the second PSNE of this game.
Table II: (a) Joint probability distribution over strategies $a_{1}$ and $a_{2}$ under correlated equilibrium. (b) An example payoff matrix for a 2-player game.

\begin{tabular}{|c|c|c|}
\cline { 2 - 3 } \multicolumn{1}{c|}{$(a)$} & $\boldsymbol{a}_{\boldsymbol{1}}$ & $\boldsymbol{a}_{2}$ \\
\hline $\boldsymbol{a}_{1}$ & $p_{1,1}$ & $p_{1,2}$ \\
\hline $\boldsymbol{a}_{2}$ & $p_{2,1}$ & $p_{2,2}$ \\
\hline
\end{tabular}

\begin{tabular}{|c|c|c|}
\cline { 2 - 3 } \multicolumn{1}{c|}{$(b)$} & $\boldsymbol{a}_{1}$ & $\boldsymbol{a}_{2}$ \\
\hline $\boldsymbol{a}_{1}$ & 0,0 & 9,7 \\
\hline $\boldsymbol{a}_{2}$ & 7,9 & 0,0 \\
\hline
\end{tabular}

Definition 2: The mixed strategy Nash Equilibrium [12] of the spectrum sharing game is a probability distribution $\hat{p}$ over the set of actions $A$ for player $i$ such that

$$
\hat{p}=\left\{p_{1}, p_{2}, \ldots p_{k}\right\} \in \mathbb{R}_{\geq 0}^{k} \text {, and } \sum_{j=1}^{k} p_{j}=1
$$

which makes the opponents indifferent about the choice of their strategies by making the payoffs from all of their strategies equal.

Let $\alpha$ be the probability with which player 1 plays strategy $a_{k}$ and $\beta=(1-\alpha)$ be the probability of playing strategy $a_{j}$, then from the payoffs of table II, the expected utility of player 2 for playing strategy $a_{k}$ is given by:

$$
E U_{2}\left(a_{k}\right)=\alpha u\left(a_{k}, a_{k}\right)+\beta u\left(a_{j}, a_{k}\right)
$$

Similarly, the expected utility of player 2 for playing strategy $a_{j}$ is given by:

$$
E U_{2}\left(a_{j}\right)=\alpha u\left(a_{k}, a_{j}\right)+\beta u\left(a_{j}, a_{j}\right)
$$

According to definition 2, player 2 will be indifferent about the choice of strategies when the expected utilities from playing strategies $a_{k}$ and $a_{j}$ are equal, i.e.,

$$
E U_{2}\left(a_{k}\right)=E U_{2}\left(a_{j}\right),
$$

Substituting (2) and (3) in (4), we have:

$$
\begin{gathered}
\beta u_{k}=\alpha u_{j} \\
\Rightarrow \alpha=\frac{u_{k}}{u_{k}+u_{j}} \quad \text { and } \quad \beta=1-\alpha=\frac{u_{j}}{u_{k}+u_{j}}
\end{gathered}
$$

Therefore, the MSNE for the spectrum sharing game is given by the distribution $\hat{p}=\{\alpha, \beta\}$ which means that when both players select strategies $a_{k}$ and $a_{j}$ with probabilities $\alpha$ and $\beta$ respectively, then their opponents will be indifferent about the outcomes of the play and mixes for its choice of strategy. We discuss the efficiency and fairness of PSNE and MSNE after deriving $\mathrm{CE}$ for our spectrum sharing game in the next subsection.

\section{Centralized Correlated Equilibrium for 2-Player Game}

Under pure and mixed strategy Nash Equilibria, it is assumed that the players choose their strategies independently without any communication. However as we demonstrate next, it is in every player's interest to coordinate their actions such that the outcomes are favorable to all players by avoiding 
ending up on the same channels, thereby making it an anticoordination game. Such coordination can be achieved with a trusted central entity that can provide an external recommendation signal which can be either public or private signals or it can be learnt for distributed implementation without the need for a central entity. In this subsection, we present the centralized algorithm to achieve $\mathrm{CE}$ while the distributed algorithm is presented in the next subsection.

$\mathrm{CE}$ is a state when given the availability of an external recommendation signal, none of the players can achieve a greater payoff by ignoring that signal when all other players follow the recommended action. Formally, CE is defined as:

Definition 3: A probability distribution is a correlated equilibrium of a game when [13]:

$$
\sum_{a_{-i} \in A_{-i}} \pi\left(a_{i}, a_{-i}\right)\left[u_{i}\left(a_{i}, a_{-i}\right)-u_{i}\left(a_{i}^{\prime}, a_{-i}\right)\right] \geq 0, \quad \forall i \in N
$$

where $\pi\left(a_{i}, a_{-i}\right)$ is the joint probability distribution of players to select a certain strategy pair in the next time slot. The inequality (11) represents that selecting some different strategy $a_{i}^{\prime}$ instead of $a_{i}$ in the next time slot will not result in a higher payoff for a player given that all other players adhere to the recommended strategy. In a centralized implementation of correlated equilibrium for a 2-player 2-strategy game such as the one shown in table II, any external entity may be selected as the 'recommender', a trusted entity that calculates and provides the external recommendation signal for all contending CRNs according to the CE joint probability distribution $\pi=$ $\left(p_{1,1}, p_{1,2}, p_{2,1}, p_{2,2}\right)$. The strategic form of such a correlated strategy pair is shown in table II (a). A correlated strategy pair means that the action pair $\left(a_{1}, a_{1}\right)$ is played with probability $p_{1,1}$ and action pair $\left(a_{1}, a_{2}\right)$ is played with probability $p_{1,2}$ etc.

Here we investigate the centralized CE using linear optimization approach. The objective function $J$ to find the optimal strategy CE for a 2-player game can be defined as:

$$
J=\max _{p_{i, j}} \sum_{i=1}^{2} \sum_{j=1}^{2}\left[u_{1}\left(a_{i}, a_{j}\right)+u_{2}\left(a_{i}, a_{j}\right)\right] p_{i, j}
$$

where the constraints for $\mathrm{CE}$ in (12) are:

$$
\begin{aligned}
& p_{1,1}+p_{1,2}+p_{2,1}+p_{2,2}=1 \\
& p_{1,1}\left[u_{1}\left(a_{1}, a_{1}\right)-u_{1}\left(a_{2}, a_{1}\right)\right] \geq p_{1,2}\left[u_{1}\left(a_{2}, a_{2}\right)-u_{1}\left(a_{1}, a_{2}\right)\right] \\
& p_{2,1}\left[u_{1}\left(a_{1}, a_{2}\right)-u_{1}\left(a_{2}, a_{2}\right)\right] \geq p_{2,2}\left[u_{1}\left(a_{1}, a_{2}\right)-u_{1}\left(a_{1}, a_{1}\right)\right] \\
& p_{1,1}\left[u_{2}\left(a_{1}, a_{1}\right)-u_{2}\left(a_{1}, a_{2}\right)\right] \geq p_{2,1}\left[u_{2}\left(a_{2}, a_{2}\right)-u_{2}\left(a_{2}, a_{1}\right)\right] \\
& p_{1,2}\left[u_{2}\left(a_{1}, a_{2}\right)-u_{2}\left(a_{1}, a_{1}\right)\right] \geq p_{2,2}\left[u_{2}\left(a_{2}, a_{1}\right)-u_{2}\left(a_{2}, a_{2}\right)\right]
\end{aligned}
$$

For the game of table II, any correlated equilibrium of the form $\pi=(0, p, 1-p, 0)$ will maximize the sum of expected payoffs for the players because it eliminates the possibility of
Table III: No-Regret Learning Algorithm

\begin{tabular}{|l|}
\hline initialization: \\
$p_{i}^{1}\left(a_{i}\right) \leftarrow \frac{1}{k_{i}}, \forall a \in A_{i}, t \leftarrow 1$ \\
\hline loop: \\
Choose strategy $a_{i}^{t} \in A_{i}$ with probability $p_{i}^{t}\left(a_{i}\right)$ \\
Observe payoff $u_{i}^{t}$ for current time slot $t$ \\
For every player, compute regret $N R_{i}^{\tau}$ for all actions \\
$a_{i}^{\prime} \in A_{i}$ not played upto current time $t$ with $(20)$ and (21) \\
Calculate $p_{i}^{t+1}$ prob. of selecting the strategy $a_{i}$ and \\
$\left(a_{i}^{\prime}\right)$ for next time slot with (22) and (23) \\
$t \leftarrow t+1$ \\
end loop
\end{tabular}

the players contending for the same channel. For an egalitarian equilibrium which is fair and maximizes the sum of expected payoffs, we have an additional constraint such that:

$$
p_{1,2}=p_{2,1} \text { and } p_{1,1}=p_{2,2}=0
$$

Having the recommender to provide external signal based on (12) and the constraints (13) - (18) ensures that probability of the two players ending up in the same channel is minimized so that the spectrum opportunity is not wasted and players' payoffs can be maximized. Furthermore, it must be noted that the external recommendation signal is not binding and players are free to ignore recommended actions.

Consider a situation in which the recommender selects an egalitarian CE probability distribution $\pi=(0,1 / 2,1 / 2,0)$ over the payoff matrix of table II (b) in order for the two players to avoid selecting the strategy pairs $\left(a_{1}, a_{1}\right)$ and $\left(a_{2}, a_{2}\right)$. The external signal recommends player 1 to select action $a_{2}$ i.e., channel 2 which is of lower quality and results in a smaller payoff of 7 compared with a payoff of 9 if channel 1 was selected for next time slot. Player 1 knows that player 2 will follow the recommended action because it has been recommended a higher quality channel. It is however in player 1 's interest to select the action recommended by the external signal since it would yield a higher payoff of 7 instead of 0 if external signal is ignored and both players end up selecting the same higher quality channel.

CE can be implemented in a similar manner as equations (12)-(18) for a multi-player game using linear programming; however, the number of constraints grows exponentially with the number of players and their strategies and the problem grows at a polynomial rate [14]. We omit the discussion of centralized correlated equilibrium for $N$-player game due to space limitation.

\section{Distributed Correlated Equilibrium for N-Player Game}

In this subsection we utilize the No-Regret learning algorithm to achieve CE [15] in CRNs in a multi-player scenario without the need of having a trusted entity to act as a centralized/external recommendation signal provider. No- 
regret learning algorithm is based on the concept of minimizing a player's regret in the hindsight for not playing other strategies in every time slot up to the current time $t$.

Specifically, suppose that the game is played repeatedly at every time slot $t=1,2,3, \ldots$ and given a history of play $h_{t}=\left(a_{i}^{\tau}\right)_{\tau=1}^{t}$ up to time $t$, every player $i$ chooses a probability $p_{i}^{t+1} \in \pi\left(A_{i}\right)$ of selecting the same strategy $a_{i}^{\tau}$ for the next time slot. The probability for selecting a strategy for the next time slot is calculated as follows: For every two different strategies $a_{i}^{\prime}$ and $a_{i} \in A_{i}$ up to time $t$, if player $i$ replaces strategy $a_{i}$ with strategy $a_{i}^{\prime}$ every time that it was played then the payoff for time $\tau$ will become:

$$
\omega_{i}^{\tau}\left(a_{i}^{\prime}\right)= \begin{cases}u_{i}^{\tau}\left(a_{i}^{\prime}, a_{-i}^{\tau}\right) & \text { if } a_{i}^{\tau}=a_{i} \\ u_{i}^{\tau}\left(a_{i}^{\tau}\right) & \text { otherwise }\end{cases}
$$

Then the average difference in player $i$ 's payoff up to time $t$ is given by:

$$
\delta_{i}^{t}\left(a_{i}^{\prime}, a_{i}\right)=\frac{1}{t} \sum_{\tau=1}^{t}\left[\omega_{i}^{\tau}\left(a_{i}^{\prime}\right)-u_{i}^{\tau}\left(a_{i}^{\tau}\right)\right]
$$

and player $i$ 's average regret at time $t$ is given by:

$$
N R_{i}^{t}\left(a_{i}^{\prime}, a_{i}\right)=\left[\delta_{i}^{t}\left(a_{i}^{\prime}, a_{i}\right)\right]^{+}
$$

and the probability of playing the strategies $a_{i}$ and $a_{i}^{\prime}$ in the next time slot is a function of a player's average regret and is given by:

$$
\begin{aligned}
& p_{i}^{t+1}\left(a_{i}^{\prime}\right)=\frac{1}{\mu} N R_{i}^{\tau}\left(a_{i}^{\prime}, a_{i}\right) \\
& p_{i}^{t+1}\left(a_{i}\right)=1-p_{i}^{t+1}\left(a_{i}^{\prime}\right)
\end{aligned}
$$

The parameter $\mu>2 M_{i}(k-1)$, such that $k$ is the number of channels and $M_{i}$ is the upper bound on $\left|u_{i}().\right|$. Its value is independent of time as well as the play's history and also ensures that there is always a positive probability of staying in the same channel as in the previous time slot. As $t \rightarrow \infty$, the empirical probability distribution $\pi$ over the $N$-tuples of strategies converges to the CE [14]. A summary of the NoRegret learning algorithm is given in table III.

\section{E. Discussion}

Here we provide a discussion on the fairness and efficiency of pure and mixed strategy Nash equilibria compared with CE. Consider the payoff matrix of table II(b) in which gaining access to channel 1 brings a payoff of 9 to the CRN while being of comparatively lower quality, channel 2 brings a payoff of 7. There are two pure strategy Nash equilibria for this anti-coordination game: $\left(a_{1}, a_{2}\right)$ and $\left(a_{2}, a_{1}\right)$, however both of them are unfair because one player always gets a smaller payoff than the other.

In order to be fair, the expected utility for both players must be equal and to achieve that, both players would have to mix their strategies in a way that their opponent is indifferent about

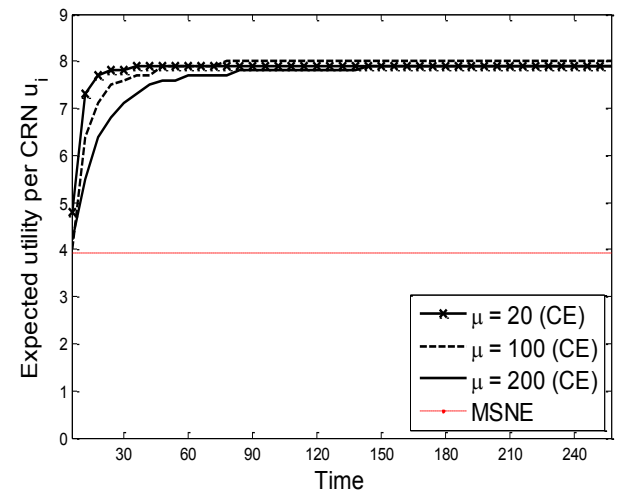

Figure 1: Comparison of MSNE and CE at different values of the inertia parameter $\mu$. Different values of $\mu$ achieve the same convergence value of expected utility however at different rates.

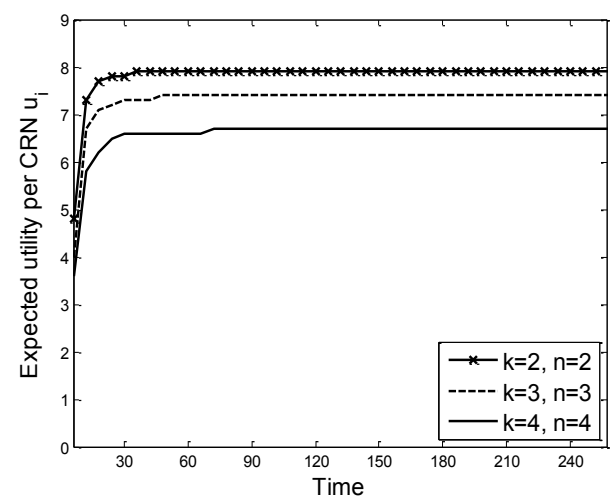

Figure 2: Comparison of CE at different values of the number of networks and channels where $n=k$. With every additional CRN, a lower quality channel was added to the spectrum resulting in smaller expected utility per CRN.

the play's outcome. The result would be the MSNE and as elaborated in section III.B above, the MSNE for the game in table $\mathrm{II}(\mathrm{b})$ is the distribution $(9 / 16,7 / 16)$ over the set of strategies for both players. When the players mix their strategies according to MSNE, then each player gains an expected utility equal to $(9 / 16 \times 7 / 16)(9+7)=3.93$ according to equations (2)-(4). This expected utility is even smaller than the payoff of the lower quality channel i.e., 7. This means that a player will always do better than the MSNE even if it always selected the lower quality channel. Furthermore, there is always a $(9 / 16 \times 9 / 16)+(7 / 16 \times 7 / 16)=50.7 \%$ chance of both players ending up in the same channel. Clearly MSNE is an inefficient solution to the anti-coordination game.

$\mathrm{CE}$ for the game of table $\mathrm{II}(\mathrm{b})$ can be calculated by equation (12) under the constraints of inequalities (13) - (18) which is the joint probability distribution of $\pi=(0,1 / 2,1 / 2$, 0 ) for the four action pairs $\left(a_{1}, a_{1}\right),\left(a_{1}, a_{2}\right),\left(a_{2}, a_{1}\right)$, and $\left(a_{2}, a_{2}\right)$. Expected utility for both players from the CE of this game is $1 / 2 \times(9+7)=8$, which is greater than the payoff from PSNE and MSNE as well as the lower quality channel. This proves that $\mathrm{CE}$ is fair and efficient at the same time and it also maximizes the minimum expected utility of all players. 


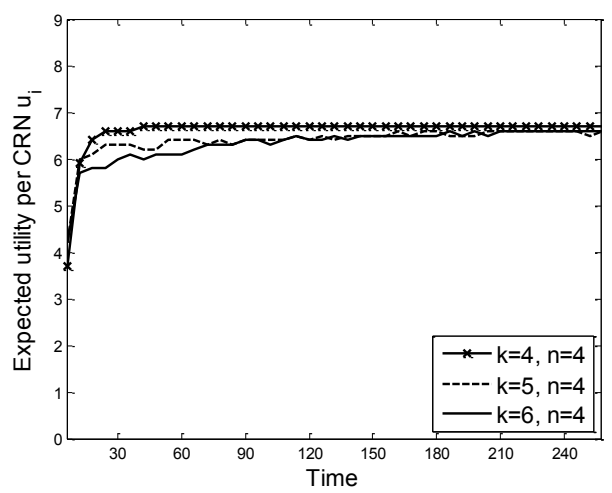

Figure 3: Comparison of CE when $k \geq n$. CRNs always select the best out of the available pool of channels therefore the convergence value of expected utilities are equal however convergence rate increase as $n \rightarrow k$.

\section{SIMULATION MODEL AND RESULTS}

\section{A. Simulation Setup}

For the purpose of validating the game model, we implemented our proposed anti-coordination game along with the No-Regret learning algorithm. We verify that $\mathrm{CE}$ is achievable, fair and efficient as it always yields a higher expected utility per CRN as compared with MSNE. For the purpose of simulation, $n$ represents the number of CRNs and $k$ is the number of channels of the spectrum available for secondary access by the CRNs. We first carry out the comparison of $\mathrm{CE}$ and MSNE with a 2-player 2-channel game i.e., $n=2$ and $k=2$ and calculate expected utilities per CRN. Later we carry out simulations with varying number of players and channels and demonstrate that the game always converges to CE. Since the No-Regret algorithm of all CRNs approaches CE based solely on a given network's own payoff observations, it allows the distributed implementation of our proposed anti-coordination game. Inertia parameter of the NoRegret learning algorithm is $\mu$ whose value is kept constant except for figure 1 .

\section{B. Simulation Results}

Figure 1 shows a comparison of expected utilities per CRN under MSNE and $\mathrm{CE}$ with various values for the inertia parameter $\mu$. Payoff value for channel-1 is 9 while channel-2 has a payoff of 7 . Compared with all the three plots for $\mathrm{CE}$ where the expected utilities converge to 8 per CRN, MSNE yields a smaller expected utility of 3.93 per CRN, proving our analysis that $\mathrm{CE}$ is more efficient than MSNE. Different values of $\mu$ achieve $\mathrm{CE}$ at different rates however the convergence values are identical. As evident from figure 1, the parameter $\mu$ reflects a CRN's propensity towards staying in the same channel in next time as the previous one.

Figure 2 shows different values for CE while increasing the number CRNs as well as the number of channels such that $n$ is always equal to $k$. The channels added to the spectrum for contention are always of lower quality than the existing

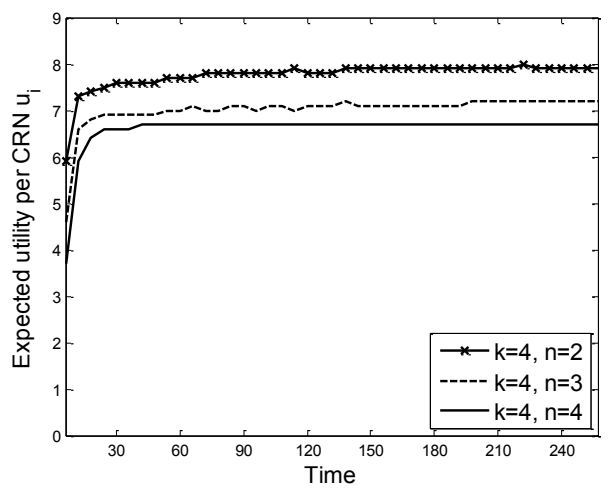

Figure 4: Comparison of CE when $n \leq k$. With a fixed value of $k$, increase in $n$ causes a decrease in the expected utilities however convergence rate increase as $n \rightarrow k$.

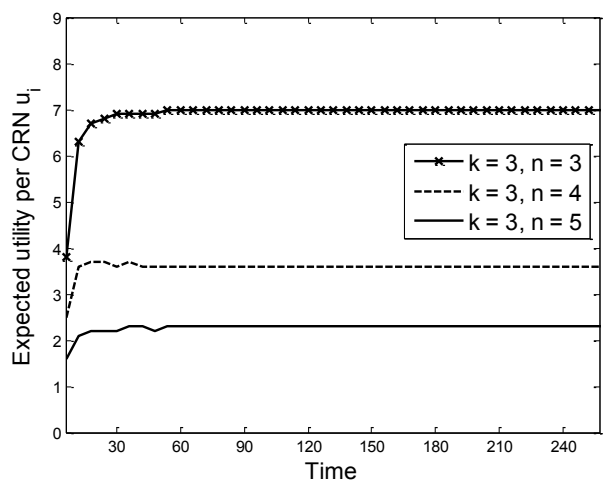

Figure 5: Comparison of CE when $n \geq k$. Every time slot will have at least one collision when $n>k$ thereby decreasing the expected utilities per CRN to drop significantly.

channels which is why the expected utility per CRN always decreases for every increase in the number of available channels from $k=2$ to $k=4$. Payoff values for channels 1 through 4 are 9, 7, 6 and 5 respectively. As can be seen from figure 2 , the rate of convergence slows down as the number of networks and channels is increased.

Figure 3 shows the CE for expected utilities per CRN over time such that $k \geq n$ i.e., increasing the number of available channels from 4 to 6 while keeping the number of contending CRNs constant at 4 . Notice that the convergence value for expected utility is the same for all cases. It shows a very important aspect of the No-Regret algorithm which allows CRNs to always have a fair distribution of channel resources as players choose the highest quality channels from the pool of available channels. Also, the speed of convergence to $\mathrm{CE}$ is fastest when the number of CRNs is equal to the number of available channels i.e., $n=k$. Payoff values for channels 1 through 6 are kept at 9, 7, 6, 5, 4 and 3 respectively.

Figure 4 shows the CE for expected utilities per CRN over time such that $n \leq k$ i.e., increasing the number of CRNs from 2 to 4 while keeping the number of available channels constant at 4 . Intuitively, expected utility per CRN is lowest at $n=4$ and $k=4$ as compared with the situation when the number of contending networks is smaller however, the speed of 
convergence to $\mathrm{CE}$ in figure 4 is fastest when $n=k$. Payoff values for channels 1 through 4 are 9, 7,6 and 5 respectively.

Finally, figure 5 shows the results of simulation when $n \geq$ $k$. It shows that as soon as the number of networks contending for channels becomes more than the number of channels available, there will always be at least one collision between two or more CRNs in every time slot making the expected utility per CRN to drop significantly. However, the No-Regret algorithm still manages to achieve CE despite much degraded expected utilities per CRN.

\section{CONCLUSIONS}

Coexistence protocols employed by collocated CRNs usually do not take into consideration the fact that spectrum bands vary significantly with regards to channel quality thereby making some channels of the spectrum bands more attractive to CRNs than others. In this paper, we aimed at solving the problem of sharing heterogeneous spectrum by adopting a game theoretic approach. We demonstrated that correlated equilibrium solves the problem of efficiency and fairness with pure and mixed strategy Nash equilibria. To address the issues associated with centralized implementation, we proposed the use of No-Regret learning algorithm that converges to correlated equilibrium in a distributed manner.

\section{REFERENCES}

[1] Taher, T.M.; Bacchus, R.B.; Zdunek, K.J.; Roberson, D.A., "Long-term spectral occupancy findings in Chicago," IEEE Symposium on New Frontiers in Dynamic Spectrum Access Networks (DySPAN), 2011.

[2] U.S. FCC, ET Docket 04-186, "Notice of Proposed Rule Making, in the matter of Unlicensed Operation in the TV Broadcast Bands," May 25, 2004.

[3] IEEE 802.22 2011 Standard for Wireless Regional Area Networks in TV Whitespaces, http://www.ieee.org/22

[4] Google, Inc.'s TV Bands Database System for Operation, ET Docket No. 04-186 http://www.google.com/get/spectrumdatabase/channel/

[5] Show My White Space -TVWS database from Spectrum Bridge Inc. http://whitespaces.spectrumbridge.com/whitespaces/home.aspx

[6] Wang, B.; Han, Zhu.; Liu, K.J.R., "Peer-to-peer file sharing game using correlated equilibrium," 43rd Annual Conference on Information Sciences and Systems, IEEE CISS 2009.

[7] Sengupta, S.; Chandramouli, R.; Brahma, S.; Chatterjee, M., "A game theoretic framework for distributed self-coexistence among IEEE 802.22 networks," IEEE GLOBECOM 2008.

[8] Etkin, R.; Parekh, A.; Tse, D., "Spectrum sharing for unlicensed bands," IEEE Journal on Selected Areas in Communications (JSAC), vol.25, no.3, pp.517,528, April 2007.

[9] Jiang, C.; Chen, Y.; Gao, Y.; Liu, K.J.Ray, "Joint Spectrum Sensing and Access Evolutionary Game in Cognitive Radio Networks," IEEE Transactions on Wireless Communications, vol.12, no.5, pp.2470,2483, May 2013.

[10] Sengupta, S.; Brahma, S.; Chatterjee, M.; Shankar, N.; "Self-coexistence among interference-aware IEEE 802.22 networks with enhanced airinterface", Pervasive and Mobile Computing, Volume 9, Issue 4, August 2013.

[11] Han, Z.; Pandana, C.; Liu, K.J.R., "Distributive Opportunistic Spectrum Access for Cognitive Radio using Correlated Equilibrium and No-Regret Learning," Wireless Communications and Networking Conference, IEEE WCNC 2007

[12] Fudenburg. D.; Tirole. J.; “Game Theory”, The MIT press, 1991.
[13] Aumann, R. J.; "Correlated equilibrium as an expression of Bayesian rationality”, Econometrica, vol. 55, no. 1, pp. 1 - 18, January 1987.

[14] Papadimitriou, C. H.; Roughgarden, T.; "Computing correlated equilibria in multi-player games." Journal of the ACM 55(3), August 2008.

[15] S. Hart and A. Mas-Colell, "A simple adaptive procedure leading to correlated equilibrium", Econometrica, vol. 68, no. 5, pp. 1127-1150, September 2000. 